\title{
COVID-19 qPCR testing in women admitted for delivery in Spain: Is universal testing worthy?: A commentary
}

\author{
A. M. Cubo ${ }^{1} \cdot$ A. Villalba-Yarza ${ }^{1} \cdot$ M. V. Lapresa Alcalde ${ }^{1}$ F. J. Goenaga ${ }^{1} \cdot$ V. García-Mínguez ${ }^{1} \cdot$ M. J. Doyague ${ }^{1}$ \\ J. M. Sayagues ${ }^{2} \mathbb{D}$
}

Received: 25 June 2020 / Accepted: 24 August 2020 / Published online: 6 September 2020

○) Springer-Verlag GmbH Germany, part of Springer Nature 2020

Keywords Covid-19 $\cdot$ SARS-CoV-2 $\cdot$ qPCR test $\cdot$ Women admitted for delivery

\section{Commentary}

The current coronavirus disease 2019 (Covid-19) pandemic, caused by the severe acute respiratory syndrome coronavirus 2 (SARS-CoV-2) is expanding globally becoming a so serious public health emergency that drastic measures across all continents, including nationwide lockdowns and border closures have been necessary to slow down the spreading of the disease. Pregnant women and their fetuses represent a high-risk population during infectious disease outbreaks and they are a challenge in terms of health care [1].

Spain has been one of the most affected countries by the pandemic, with more than 243,000 positive cases and 27,000 deaths at the end of May [2]. Castilla y León has been the third with the highest number of cases $(19,104$ infections and 1928 deaths on June 11th) among the 17 autonomous communities of Spain. Of the nine provinces that make up the Community of Castilla y León, Salamanca is the second both in number of infections (4280) and deaths (369, 19\% of the whole Community) [3].

Salamanca University Hospital is one of the largest hospitals in the Community, with 2000 deliveries per year on average. From the beginning of the pandemic, testing with nasopharyngeal swabs and a quantitative polymerase-chainreaction (qPCR) was performed in the Emergency Department on patients with Covid-19 disease symptoms and an outpatient program was implemented to trace positive

A. M. Cubo

amcubo@saludcastillayleon.es

1 Department of Obstetrics and Gynecology, Salamanca University Hospital and IBSAL, Paseo San Vicente 58-182, 37007 Salamanca, Spain

2 Department of Pathology, Salamanca University Hospital and IBSAL, Salamanca, Spain patient's contacts. As evidence on the existence of a large number of asymptomatic positive patients who could act as carriers was reported [4, 5], the Obstetrics Department asked the qPCR test to be performed on all patients admitted both for delivery and to the obstetrics ward, to guarantee admission in a safe environment and to reduce the chance of transmission to other patients and to the healthcare staff.

From 23/03, the qPCR test was carried out to detect SARS-CoV-2 in all women admitted for delivery. Since then there have been 366 deliveries up to 11/06. Of these, 25 patients $(15 \%)$ have tested positive for the virus. Twelve of them were detected by qPCR on admission for delivery being all of them asymptomatic. It should be noted that two of these patients were referred from a private centre where serological screening with IgM-positive antibodies was carried out. On admission, both women tested negative for qPCR; however, considering the chance of being either Covid-19 early stage or qPCR false negative, they were treated as if they were positive [6]. qPCR test was repeated a week later becoming positive in one of them. The reasons for admission in these patients were all obstetric: six were admitted in labour, two presented premature rupture of membranes at term (PROM), two had preterm premature rupture of membranes (PPROM), one postterm pregnancy was admitted for induction and one term pregnancy presented severe pre-eclampsia. Another nine patients were detected through the outpatient contact tracing program. Six of them were asymptomatic whilst three had mild symptoms. One of the patients in this group who was positive at 31 weeks gestation and whose qPCR was negative at 33 weeks was admitted with an intrauterine fetal death and required an emergent caesarean section due to severe preeclampsia and disseminated intravascular coagulation. Four patients were tested by the hospital Emergency Department being all symptomatic: two of them had mild symptoms and 


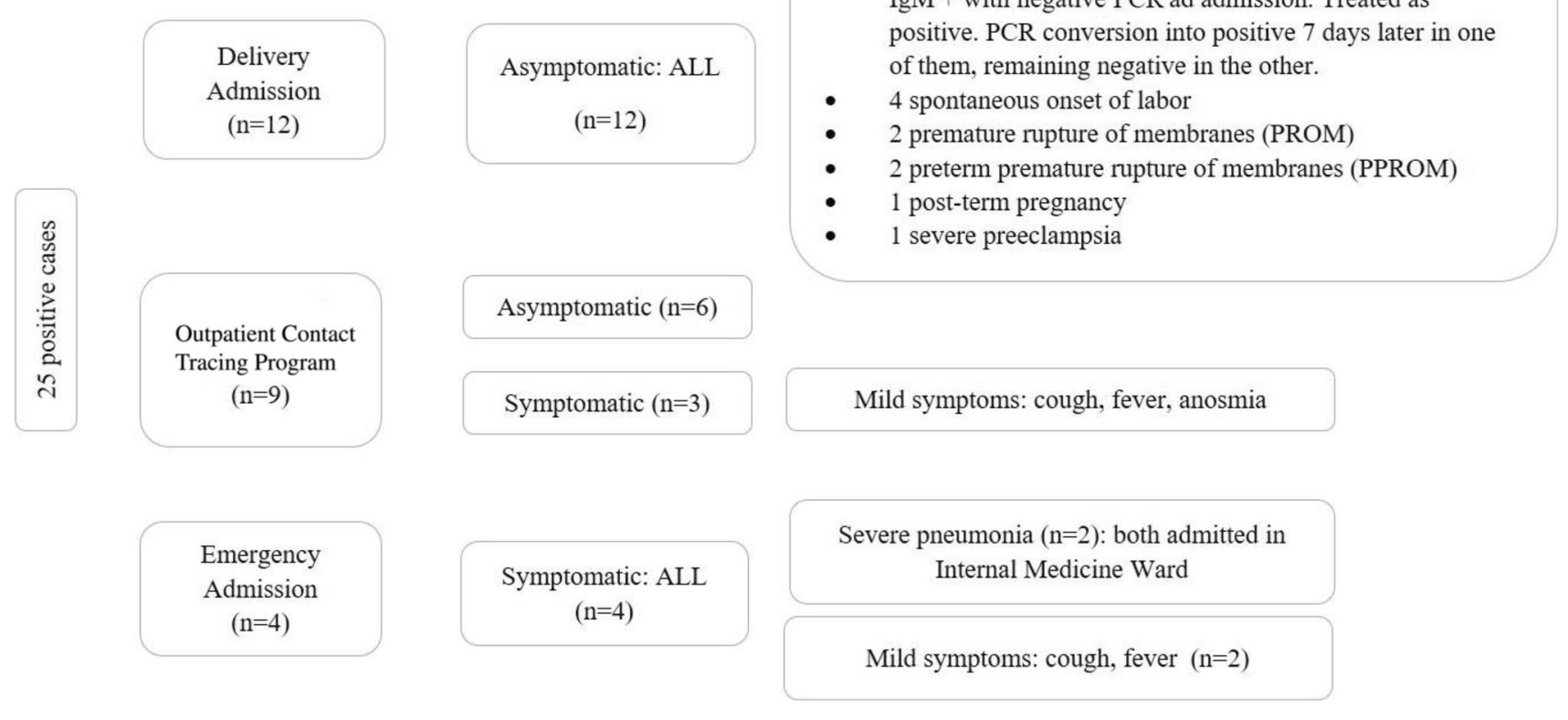

2 patients admitted for labor, referred from private hospital, IgM + with negative PCR ad admission. Treated as positive. PCR conversion into positive 7 days later in one of them, remaining negative in the other.

4 spontaneous onset of labor

2 premature rupture of membranes

- 1 post-term pregnancy

1 severe preeclampsia

Fig. 1 Clinical characteristics of the 25 patients (15\% of the total cases studied) admitted for delivery and tested positive for qPCR

were discharged under home isolation and contact tracing recommendations. The other two presented severe pneumonia being admitted to the Internal Medicine Ward. One of them developed a threatened preterm labor resistant to tocolysis, which ended in preterm delivery at 32 weeks gestation. The other patient suffered a severe worsening and required admission to Intensive Care Unit for a week (Fig. 1).

In our experience, $72 \%$ of the positive patients were asymptomatic $(n=18)$ and $20 \%$ presented mild symptoms that allowed outpatient control, without hospital admission $(n=5)$. Only $8 \%(n=2)$ required admission for severe symptoms, mainly pneumonia. These data are important, as without a prior screening test we could be facing a positive patient unaware, thus increasing the risk of transmission and therefore the spread of infection [7].

From the beginning of the crisis, admission protocols in the Obstetric Department were adapted to avoid the separation of the positive mother from her child and to guarantee, on the one hand, the isolation from the rest of the women in the ward, preventing the spread of infection to the negative patients, and on the other hand, the necessary intimacy and comfort for both mother and new-born. The implementation of qPCR testing in a universal basis has been a key factor on this point. None of the new-borns developed Covid-19 symptoms or required admission for this reason.

Spain has also been one of the countries with the highest number of infected healthcare staff to date and where the shortage of protective equipment has been particularly noticeable during the most serious moments of the crisis. In our department, almost $20 \%$ of the medical staff has been infected during the pandemic (5/26). Although it is not possible to specify, it is very likely that most of these infections had occurred during the time prior to the declaration of the state of alert and the routine performance of screening tests, with patients being attended without adequate protective measures [8]. This is particularly important not only because of the loss of health care capacity for the population as the effective number of available health care workers is reduced, but also because, if these infected workers are themselves asymptomatic, they can act as carriers of the disease and spread it further $[4,9]$.

For all the above reasons, universal Covid-19 screening in pregnant women prior to admission for delivery is beneficial not only for the women themselves, who will get more appropriate care, but also to reduce the risk of exposure of the healthcare staff, to trace the Covid-19 status in her contacts and relatives and to implement measures to slow down the spread of the pandemic.

Author contributions CAM conceived the idea and wrote the manuscript. VYA is the principal investigator of the study. LAMV, GFJ, GMV, DMJ and SJM were directly involved in the conception of this study as well as in editing the manuscript. All authors approved the final version of the manuscript.

Funding This work has been partially supported by grant from the Gerencia Regional de Salud de Castilla y León, Valladolid, Spain (GRS2045/A/19). 


\section{Compliance with ethical standards}

Conflict of interest The authors declare no potential conflicts of interest with respect to the research, authorship and publication of this article.

Institutional ethics committee The study was reviewed and approved by the ethics committee of the University Hospital of Salamanca, Salamanca, Spain.

\section{References}

1. Narang K, Ibirogba ER, Elrefaei A, Trad ATA, Theiler R, Nomura $\mathrm{R}$ et al (2020) SARS-CoV-2 in pregnancy: a comprehensive summary of current guidelines. J Clin Med 9(5):1521

2. Ministerio de Sanidad, Consumo y Bienestar Social—Professionals-Enfermedad por nuevo coronavirus, COVID-19. Available from: https://www.mscbs.gob.es/en/profesionales/saludPublica/ ccayes/alertasActual/nCov-China/home.htm. Accessed 11 June 2020

3. Explore-Análisis de datos abiertos JCyL. Available from: https ://analisis.datosabiertos.jcyl.es/explore/?refine.theme $=$ Salud \&sort $=$ modified. Accessed 11 June 2020
4. Yu X, Yang R (2020) COVID-19 transmission through asymptomatic carriers is a challenge to containment. Influenza Other Respi Viruses 14:474-475

5. Gandhi M, Yokoe DS, Havlir DV (2020) Asymptomatic transmission, the achilles' heel of current strategies to control COVID-19. N Engl J Med 382:2158-2160

6. Woloshin S, Patel N, Kesselheim AS (2020) False negative tests for SARS-CoV-2 infection-challenges and implications. N Engl J Med 383(6):e38

7. Sutton D, Fuchs K, D'Alton M, Goffman D (2020) Universal screening for SARS-CoV-2 in women admitted for delivery. $\mathrm{N}$ Engl J Med 382(22):2163-2164

8. Lancet T (2019) COVID-19: protecting health-care workers. Lancet 395:922

9. Bai Y, Yao L, Wei T, Tian F, Jin DY, Chen L et al (2020) Presumed asymptomatic carrier transmission of COVID-19. JAMA 323:1406-1407

Publisher's Note Springer Nature remains neutral with regard to jurisdictional claims in published maps and institutional affiliations. 